\section{Detection of Helicobacter Pylori in the lacrimal sac mucosa of the patients with primary acquired nasolacrimal duct obstruction}

\author{
Naser Owji, ${ }^{1}$ \\ Seyed Mohammad Bagher Abtahi, ${ }^{2}$ \\ Negar Azarpira ${ }^{3}$ \\ 'Poostchi Eye Research Center; \\ 2Department of Ophthalmology, Khallili \\ Hospital; ${ }^{3}$ Organ Transplant Research \\ Center, Shiraz University of Medical \\ Sciences, Shiraz, Iran
}

\begin{abstract}
Helicobacter pylori have been detected in sinonasal mucosa in both normal and pathologic condition. The nasolacrimal duct is withble. The present investigation was planned to determine the presence of $H$. pylori by polymerase chain reaction (PCR) in the nasal and lacrimal sac mucosa in patients with primary acquired nasolacrimal duct obstruction who underwent dacryocystorhinostomy.
\end{abstract} in the medial wall of maxillary sinus and open into the nasal cavity, so ascending colonization of nasolacrimal duct and lacrimal sac is possible. The aim of this study is to investigate the presence of $H$. pylori by polymerase chain (PCR) reaction in the nasal and lacrimal sac mucosa of the patients with primary acquired nasolacrimal duct obstruction. Eighty patients with primary acquired nasolacrimal duct obstruction who were scheduled for dacryocystorhinostomy enrolled in the study. The patients were asked if they suffered from the classic symptoms of gastroesophageal reflux disease (heart burn, regurgitation, and acid taste). Tissue samples from the lacrimal sac mucosa and nasal mucosa were obtained during dacryocystorhinostomy surgery. The tissues were analyzed for detection of $H$. pylori DNA by PCR. The mean age of patients was 41.96 \pm 14.7 years (age range, 17-84 Years). PCR for $H$. pylori DNA was positive in the nasal mucosa in 3 patients, in the lacrimal sac mucosa in 2 patients and in both nasal mucosa and lacrimal sac mucosa in 1 patient. Classic symptoms of gastroesophageal reflux disease were found in 16 patients (20\%). It is possible to detect $H$. pylori in the lacrimal sac mucosa of some patients with primary acquired nasolacrimal duct obstruction. More comprehensive studies are needed to determine whether H. pylori plays an etiopathologic role in the development of primary acquired nasolacrimal duct obstruction.

\section{Introduction}

Helicobacter pylori is a microaerophilic Gram-negative spiral organism that normally inhabits the gastric mucus layer. $H$. pylori is a major cause of gastritis and peptic ulcer disease and has been implicated in the development of gastric malignancy. ${ }^{1}$ Colonization of $H$. pylori has been found in dental plaques, saliva, tonsils and adenoids. ${ }^{2,3}$ Recently this organism was also detected in the nasal and maxillary sinus specimens of patients with chronic sinusitis. ${ }^{4-6}$ Some believe that the sinonasal mucosa serves as reservoir for $H$. pylori infections., ${ }^{5,6}$ The nasolacrimal duct is within the medial wall of the maxillary sinus and opens into the nasal cavity, so ascending colonization of nasolacrimal duct and lacrimal sac is possi-

\section{Materials and Methods}

Eighty consecutive patients with primary acquired nasolacrimal duct obstruction were enrolled. They were scheduled for dacryocystorhinostomy (DCR) in Khallili Teaching Hospital (Shiraz, Iran). The study was approved by the local ethics committee (Poostchi Eye Research Center). All patients gave their informed consent before enrolling in the study. All patients were examined by an otolaryngologist to rule out possible secondary causes of nasolacrimal duct obstruction. The patients were asked whether they had classic symptoms of gastroesophageal reflux disease (heart burn, regurgitation, and acid taste) and whether they had used antibiotics, bismuth compounds, $\mathrm{H}_{2}$ receptor blockers, antacid drugs, and proton pump inhibitors before the surgery. Those who used the above systemic medications four weeks before the surgery were excluded from the study. Classic external DCR was carried out under general anesthesia. Tissue samples from lacrimal sac mucosa and nasal mucosa were prepared at the time of lacrimal sac and nasal mucosal flap preparation.

Biopsy specimens were fixed in $10 \%$ buffered formalin, embedded in paraffin, sectioned and stained with hematoxylin-eosin to evaluate the pathological findings. DNA was extracted from the sample tissues (containing approximately $10 \mathrm{mg}$ of specimen) by using a tissue DNA extraction kit (Cinnagen, Tehran, Iran), following the manufacturer's instructions. Great care was taken to avoid contami-
Correspondence: Naser Owji, Department of Ophthalmology, Khallili Hospital, Oculoplasty service, Shiraz University of Medical Sciences, Shiraz, Fars, Iran. Tel/Fax: +98.711.647.1479.

E-mail: dr_oji@yahoo.com

Key words: Helicobacter Pylori, acquired nasolacrimal duct obstruction, polymerase chain reaction, nasal mucosa, lacrimal sac mucosa.

Acknowledgements: the authors would like to thank Soraya Saky, MD for assistance in data collection, and Mohammad Javad Ashraf, MD for review of pathology specimens.

Contributions: NO, manuscript conception and design, revision and final approval; SMBA, NA, data acquisition, manuscript drafting and final approval.

Funding: financially, this study is partially supported by Organ transplant research center Shiraz university of medical sciences. There is no proprietary interest.

Received for publication: 10 April 2011.

Revision received: 5 December 2011.

Accepted for publication: 6 December 2011.

This work is licensed under a Creative Commons Attribution NonCommercial 3.0 License (CC BYNC 3.0).

(C) Copyright N. Owji et al., 2011

Licensee PAGEPress, Italy

Eye Reports 2011; 1:e14

doi:10.4081/eye.2011.e14

nation both during the sample collection step and preparation step.

A polymerase chain reaction assay with the H. pylori PCR detection kit was performed according to the manufacturer's protocol. PCR primers were targeted to an ureC gene segment of $H$. pylori. All samples were positive for $\beta$-globin as an internal control. In each run, positive and negative controls were included.

Culturing of microorganisms and PCR for other microorganisms was not performed in this investigation.

The chi-square test and independent t-test were used for statistical analysis. A $P$ value of less than 0.05 was considered to be significant.

\section{Results}

The present investigation was performed on 80 patients. There were 21 male $(26.25 \%)$ and 59 female $(73.75 \%)$ patients. The mean age of patients was $41.96 \pm 14.7$ years (age range, 1784 Years). The PCR for $H$. pylori DNA was positive in the nasal mucosa in 3 patients, in the lacrimal sac mucosa in 2 patients and in both 
nasal mucosa and lacrimal sac in 1 patient. Statistically, there was no significant difference $(\mathrm{P}=0.63)$ between the mean age of patient with positive PCR (39.16 Years) and that of the patients with negative PCR (42.18 Years). Classic symptoms of gastroesophageal reflux disease (GERD) were found in 16 patients (20\%). Classic symptoms of GERD were present in 1 PCR positive patient and in 15 PCR negative patients. There was no statistically significant difference between the two groups $(\mathrm{P}=0.65)$. There was, however, a statistically significant difference between the mean ages of the patients with and without classic symptoms of GERD, which were 50.81 years, and 39.75 years, respectively $(\mathrm{P}=0.007)$.

\section{Discussion}

H. pylori normally inhabits the gastric mucosa. $H$. pylori has been detected in dental plaque, saliva, tonsils, and adenoids. ${ }^{2,3}$ The oral cavity might be considered a reservoir for $H$. pylori. ${ }^{7}$ H. pylori has been found in nasal polyp, nasal mucosa and maxillary sinus mucosa in some patients with chronic sinusitis. ${ }^{4-6}$

The role of $H$. pylori infection in ophthalmic diseases such as chronic ocular inflammation, glaucoma,${ }^{8}$ rosacea, and chronic blepharitis ${ }^{9}$ has been postulated. The complex interaction between $H$. pylori and ocular disease is a field of ongoing research. Gastric colonization by $H$. pylori infection is associated with release of variable proinflammatory and vasoactive substances such as interleukins, tumor necrosis factor $\alpha$, interferon $\gamma$, prostaglandins, and Creactive protein. In addition, in peptic ulcer disease associated with $H$. pylori infection, nitric oxide and endothelin-1 (constrictor of arterioles and venules) are increased. These substances may influence, for example, the pathophysiology of glaucoma by microvascular disturbance of anterior optic nerve vessels and the ophthalmic artery. ${ }^{10}$ Production of oxidative stress and circulating lipid peroxides by $H$. pylori infection could affect pathophysiology of glaucoma as well. ${ }^{11}$ Similar mechanisms have been proposed for a possible association between $H$. pylori infection and choroidoretinopathy. ${ }^{12}$ Gastrin (a potent vasodilator) level in the serum is elevated in patients with $H$. pylori associated gastric disease. Circulating gastrin may cause vasodilation of the skin and ocular surface vessels leads to rosacea manifestations. ${ }^{13}$

To the best of our knowledge, this is the first study reporting the presence of $H$. pylori in the lacrimal sac mucosa. The detection of $H$. pylori in the lacrimal sac mucosa shows that colonization is not restricted to the nasal cavity, but ascending colonization via nasolacrimal duct is possible. The postulated mechanisms by which $H$. pylori reaches the nasal cavity are as follows. First, $H$. pylori may come from the stomach by gastroesophageal reflux (GER). Second, the oral cavity may act as a reservoir of H. pylori, and the microorganism may come to the nasal cavity directly through oronasal reflux.${ }^{4,6}$ In our study, the prevalence of subjective signs of GERD was similar in both PCR positive and negative patients. We did not investigate GER objectively. In addition, we did not investigate the presence of $H$. pylori in the stomach or oral cavity, so we do not know about the source of infection in these cases.

The role of $H$. pylori infection in the pathogenesis of upper respiratory system diseases has been evaluated by some investigators. ${ }^{14}$ It is speculated that there is a relationship between GERD and chronic rhinopharyngitis and rhinosinusitis in both children and adult. ${ }^{15-17}$ It is suggested that direct reflux of gastric juice into nasopharynx may cause mucosal edema and inflammation, leading to obstruction of sinus ostia. ${ }^{18}$ Recently focus has been on the possible direct role of $H$. pylori in tissue injury of the sinonasal mucosa and development of chronic rhinosinusitis., However, the causality has not been established yet.

Primary acquired nasolacrimal duct obstruction (PAND0) occurs in the absence of an obvious precipitating cause. ${ }^{19}$ Pathologic studies of lacrimal passages have indicated that PAND0 results from fibrous obstruction secondary to chronic inflammation. The first event in nasolacrimal duct obstruction is unknown but dacryostenosis develop as a result of inflammation and fibrosis. Dacryostenosis with stasis and secondary infection may lead to complete nasolacrimal duct obstruction. ${ }^{20,21}$ Descending infection from the conjunctiva has been considered a causative factor in dacryostenosis. In addition to the descending infection from the eye, ascending infection from nasal mucosa could be the starting point of dacryostenosis. ${ }^{22}$ Bacteria, fungi, and parasites have been implicated as underlying causes of lacrimal drainage obstruction. ${ }^{19}$

In our series, those who used systemic antibiotics or bismuth during the four weeks before the surgery were excluded, as the treatment may eradicate or decrease $H$. pylori presence. The lacrimal sac mucosa of three patients was positive for $H$. pylori. Retrograde colonization of nasolacrimal duct and hence lacrimal sac mucosa from the nasal cavity is possible. It may be transient colonization but infection of the nasolacrimal duct and lacrimal sac could not be ruled out. It is highly speculative, but in some patients with PAND0, $H$. pylori may cause direct nasolacrimal duct mucosa injury and chronic inflammation, leading to nasolacrimal duct stenosis. If it is true, treatment of $H$. pylori in these patients is war- ranted to prevent complete nasolacrimal duct obstruction.

Limitations of the present case series suggest several areas for further research. One of the drawbacks of our study is the lack of control group. Secondly, the small group of participants limits the ability to make generalizations and requires one to view the results with caution. Hence, there is a need for further research to evaluate the presence of $H$. pylori in larger series with a control group and to evaluate the effect of treatment for $H$. pylori in patients with positive PCR.

\section{References}

1. Hashemi MR, Rahnavardi M, Bikdeli B, Dehghani Zahedani M. H. pylori infection among 1000 Southern Iranian dyspeptic patients. World J Gastroenterol 2006;12: 5479-82.

2. Unver S, Kubilay U, Sezen OS, Coskuner T. Investigation of Helicobacter pylori colonization in adenotonsillectomy specimens by means of the CLO test. Laryngoscope 2001;111:2183-6.

3. Nabwera HM, Logan RPH. Epidemiology of Helicobacter pylori: transmission, translocation and extragastric reservoirs. J Physiol Pharmacol 1999;50:711-22.

4. Morinaka S, Ichimiya M, Nakamura H. Detection of Helicobacter pylori in nasal and maxillary sinus specimens from patients with chronic sinusitis. Laryngoscope 2003;113:1557-63.

5. Dinis PB, Subtil J. Helicobacter pylori and laryngopharyngeal reflux in chronic rhinosinusitis. Otolaryngol Head Neck Surg 2006;134:67-72.

6. Ozdek A, Cirak MY, Samim E, et al. A possible role of Helicobacter pylori in chronic rhinosinusitis: a preliminary report. Laryn-goscope 2003;113:679-82.

7. Nguyen AM, el-Zaatari FA, Graham DY. Helicobacter pylori in the oral cavity. Oral Surg Oral Med Oral Pathol Oral Radiol Endod 1995;79:705-9.

8. Galloway PH, Warner SJ, Morsherd MG, Mikelberg FS. Helicobacter pylori infection and the risk of open-angle glaucoma. Ophthalmology 2003;110:922-26.

9. Sacca SC, Pascotto A, Venturino GM, et al. Prevalence and treatment of Helicobacter pylori in patients with blepharitis. Invest Ophtalmol Vis Sci 2006;47:501-8.

10. Kountouras J, Zavos C, Chatzopoulos D. Induction of apoptosis as a proposed pathophysiological link between glaucoma and Helicobacter pylori infection. Med Hypo-theses 2004;62:378-81.

11. Kountouras J, Mylpoulos N, Chatzopoulos D, et al. Eradication of Helicobacter pylori 
may beneficial in themanagement of chronic open angle glaucoma. Arch Intern Med 2002;162:1237-44

12. Giusti C,Mauget-Faysse M. Helicobacter pylori and idiopathic central serous chorioretinopathy. Swiss Med Wkly 2004;134: 395-8.

13. Mindel JS, Rosenberg EW. Is Helicobacter pylori of interest to ophthalmologist? Ophthalmology 1997;104:1729-30.

14. Kurtaran H, Uyar ME, Kasapoglu B, et al. Role of Helicobacter pylori in pathogenesis of upper respiratory system diseases. J Natl Med Assoc 2008;100:1224-32.

15. DiBaise JK, Olusola BF, Huerter JV, Quigley EM. Role of GERD in chronic resistant sinusitis: a prospective, open label, pilot trial. Am J Gastroenterol 2002;97:843-50.

16. Bothwell MR, Parsons DS, Talbot A, et al. Outcome of reflux therapy on pediatric chronic sinusitis. Otolaryngol Head Neck Surg 1999;121:255-62.

17. Ozmen S, Yücel OT, Sinici I, et al. Nasal pepsin assay and $\mathrm{pH}$ monitoring in chronic rhinosinusitis. Laryngoscope 2008;118: $890-4$.

18. Dibaise JK, Sharma VK. Does gastroesophageal reflux contribute to the development of chronic sinusitis? A review of the evidence. Dis Esophagus 2006;19:419-24.

19. Bartley GB. Acquired lacrimal drainage obstruction: An etiologic classification system, case reports, and a review of the liter- ature. Part 1. Ophthal Plast Reconstr Surg 1992;8:243-2.

20. Linberg JV, McCormick SA. Primary acquired nasolacrimal duct obstruction: a clinicopathologic report and biopsy technique. Ophthalmology 1986;93:1055-63.

21. Mauriello JA, Palydowycz S, DeLuca J. Clinicopathologic study of lacrimal sac and nasal mucosa in 44 patients with complete acquired nasolacrimal duct obstruction. Ophthal Plast Reconstr Surg 1992;8:13-21.

22. Paulsen FP, Thale AB, Maune S, Tillman BN. New insights into the pathophysiology of primary acquired dacryostenosis. Ophthalmology 2001;108:2329-35 